\title{
Narrow band imaging of adenocarcinoma arising from ectopic gastric mucosa in the upper esophagus
}

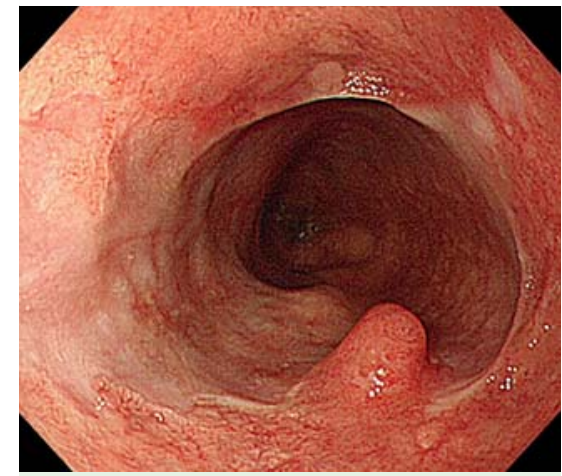

Fig. 1 Image obtained during a conventional upper gastrointestinal endoscopy in a 74-yearold man showing ectopic gastric mucosa around the entire circumference of the cervical to upper thoracic esophagus with a 7-mm elevated lesion distal to the ectopic mucosa.
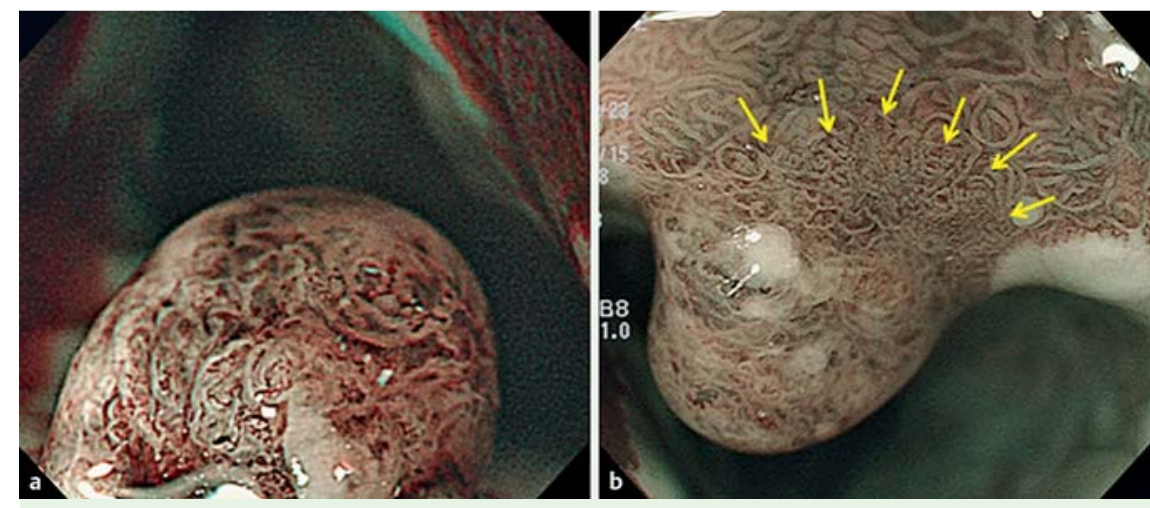

Fig. 3 Appearance on narrow band imaging (NBI) showing: a the elevated lesion with glandular structures of variable size and dense, abnormal capillaries; $\mathbf{b}$ the glandular structures at the foot of the elevated lesion, which were smaller than those in the surrounding ectopic gastric mucosa and were irregularly arranged, suggestive of tumor extension (arrows).

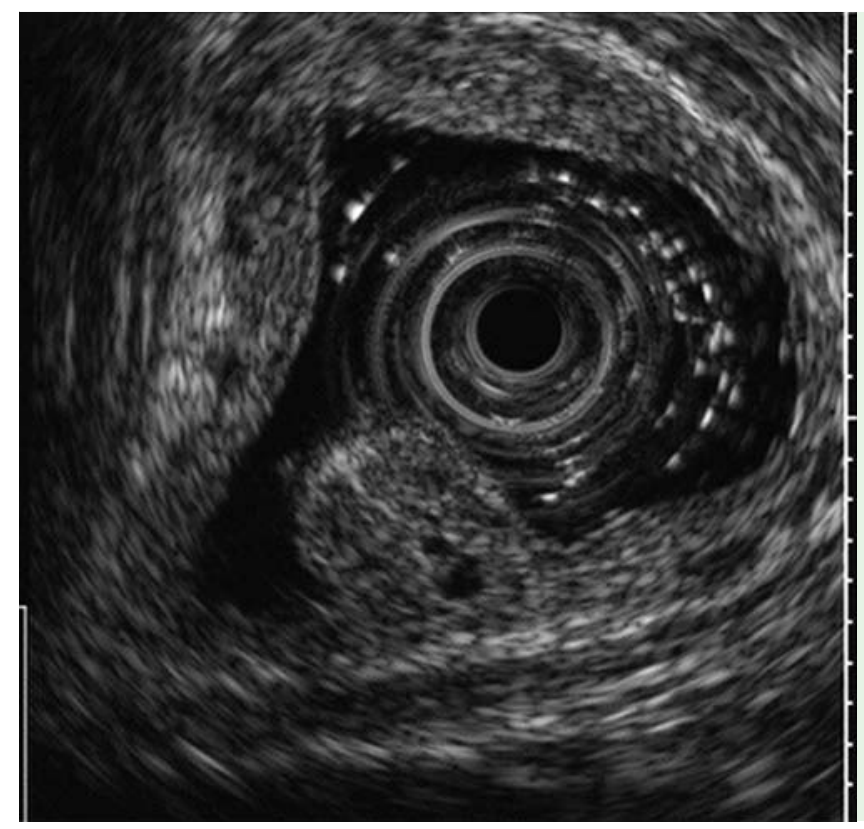

Adenocarcinomas arise rarely in the cervical and upper esophagus [1]. Among these, esophageal adenocarcinomas arising from ectopic gastric mucosa are extremely rare [2-4]. We report the first patient to undergo magnifying endoscopy with narrow band imaging (NBI) and endoscopic submucosal dissection (ESD) for an esophageal adenocarcinoma arising from ectopic gastric mucosa in the upper thoracic esophagus.

The patient was a 74-year-old man who underwent an upper gastrointestinal endoscopy for a health checkup, which revealed a circumferential area of orangered mucosa at $16-19 \mathrm{~cm}$ from the upper incisors ( Fig. 1). Conventional endoscopy of this mucosal area showed glandular structures. The site remained unstained after spraying with Lugol's solution (৫ Fig.2), which led to this being diagnosed as ectopic gastric mucosa. A 7-mm elevated lesion was also observed distal to the circumferential ectopic gastric mucosa ( Fig. 1). When viewed by magnifying endoscopy with NBI, the elevated lesion showed glandular structures of variable size and with patterns differing from those of the surrounding area and abnormal microvessels of variable diameter $($ Fig.3a). The abnormal glandular structures extended, at least in one area, to the foot of the elevated lesion, a finding suggestive of tumor extension ( $\bullet$ Fig. 3 b) There was no evidence of submucosal invasion of the tumor on endoscopic ultrasound (EUS; - Fig.4). A biopsy of the elevated lesion led to a diagnosis of adenocarcinoma. Computed tomography revealed an absence of swollen lymph nodes in the neck and mediastinum.

After informed consent had been obtained from the patient, ESD was performed. The resected specimen measured $13 \times 12 \mathrm{~mm}$ and contained a $7 \times 6-\mathrm{mm}$ tumor composed of atypical glandular structures with enlarged hyperchromatic nuclei. These structures were arranged in tubules or in irregular branching and fusion patterns and were invading the muscularis mucosae ( Fig.5a,b). Part of the specimen contained cardiac gland structures ( $\nabla$ Fig. 5 c), which on immunohistochemical staining were positive for MUC5AC and MUC6, but negative for MUC1 and MUC2 ( Fig.5d). These findings were consistent with an adenocarcinoma arising from ectopic gastric mucosa in the esophagus.

Endoscopy_UCTN_Code_CCL_1AB_2AC_3AB 


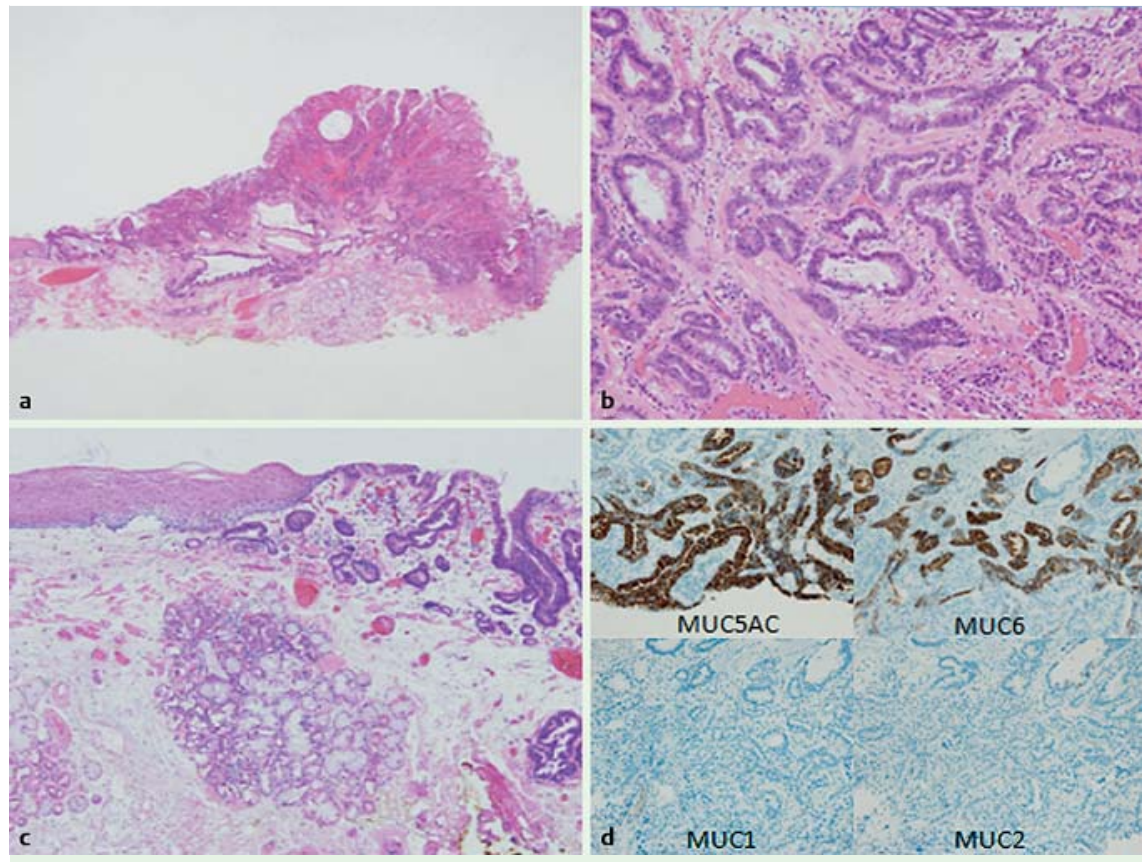

Fig.5 Appearances following hematoxylin and eosin (H\&E) staining of the specimen resected by endoscopic submucosal dissection (ESD) showing: a a $7 \times 6-\mathrm{mm}$ tumor contained within the specimen (original magnification); $\mathbf{b}$ abnormal glandular structures with, in some areas, abnormal branching patterns (magnification $\times 100$ ); $\mathbf{c}$ ectopic gastric mucosa close to the adenocarcinoma (magnification $\times 100$ ). $\mathbf{d}$ Immunohistochemical staining of the resected specimen showing that the glandular structures were positive with MUC5AC and MUC6 but negative with MUC1 and MUC2 (magnification $\times 100$ ).
Bibliography

DOI http://dx.doi.org/ 10.1055/s-0032-1325865

Endoscopy 2013; 45: E112-E113

(c) Georg Thieme Verlag KG

Stuttgart · New York

ISSN 0013-726X

\section{Corresponding author \\ K. Nonaka, MD}

Department of Gastroenterology and Hepatology Graduate School of Medical Sciences

Kumamoto University

Kumamoto 860-8556

Japan

Fax: +81-96-3710582

nonaka513@gmail.com

\section{Competing interests: None}

\section{K. Nonaka ${ }^{1}$, M. Watanabe ${ }^{2}$, H. Yuruki ${ }^{1}$, A Okuda ${ }^{1}$, K. Sakurai ${ }^{1}$, K. Iyama ${ }^{3}$, Y. Sasaki ${ }^{1}$}

${ }^{1}$ Department of Gastroenterology and Hepatology, Graduate School of Medical Sciences Kumamoto University

2 Department of Gastroenterological Surgery, Graduate School of Medical Sciences Kumamoto University

${ }^{3}$ Department of Surgical Pathology, Kumamoto University Hospital

\section{References}

1 Faintuch J, Shepard KV, Levin B. Adenocarcinoma and other unusual variants of esophageal cancer. Semin Oncol 1984; 11: $196-$ 202

2 Noguchi T, Takeno S, Takahashi $Y$ et al. Primary adenocarcinoma of the cervical esophagus arising from heterotopic gastric mucosa. J Gastroenterol 2001; 36: 704-709

3 Balon JM, Mariette C, Fabre S et al. [Primary adenocarcinoma of the cervical esophagus arising from heterotopic gastric mucosa]. Gastroenterol Clin Biol 2003; 27: 836-838

4 Akanuma N, Hoshino I, Akutsu Y et al. Primary esophageal adenocarcinoma arising from heterotopic gastric mucosa: report of a case. Surg Today, in press 2012: 10.1007/ s00595-012-0206-9 